\title{
Erratum to: Proteome Analysis of Aspergillus fumigatus Total Membrane Proteins Identifies Proteins Associated with the Glycoconjugates and Cell Wall Biosynthesis Using 2D LC-MS/MS
}

Haomiao Ouyang $\cdot$ Yuanming Luo •

Lei Zhang $\cdot$ Yanjie Li $\cdot$ Cheng Jin

Published online: 12 February 2010

(C) Springer Science+Business Media, LLC 2010

Erratum to: Mol Biotechnol

DOI 10.1007/s12033-009-9224-2

Some mistakes appear in Figures 3 and 5 in the original publication. Corrected versions of the images are reproduced here.

The online version of the original article can be found under doi: 10.1007/s12033-009-9224-2.

H. Ouyang · Y. Luo $(\bowtie) \cdot$ L. Zhang · Y. Li · C. Jin $(\bowtie)$ State Key Laboratory of Microbial Resources, Institute of Microbiology, Chinese Academy of Sciences,

A3 Datun Road, Chaoyang District, Beijing 100101, China

e-mail: luoym@im.ac.cn

C. Jin

e-mail: jinc@sun.im.ac.cn 


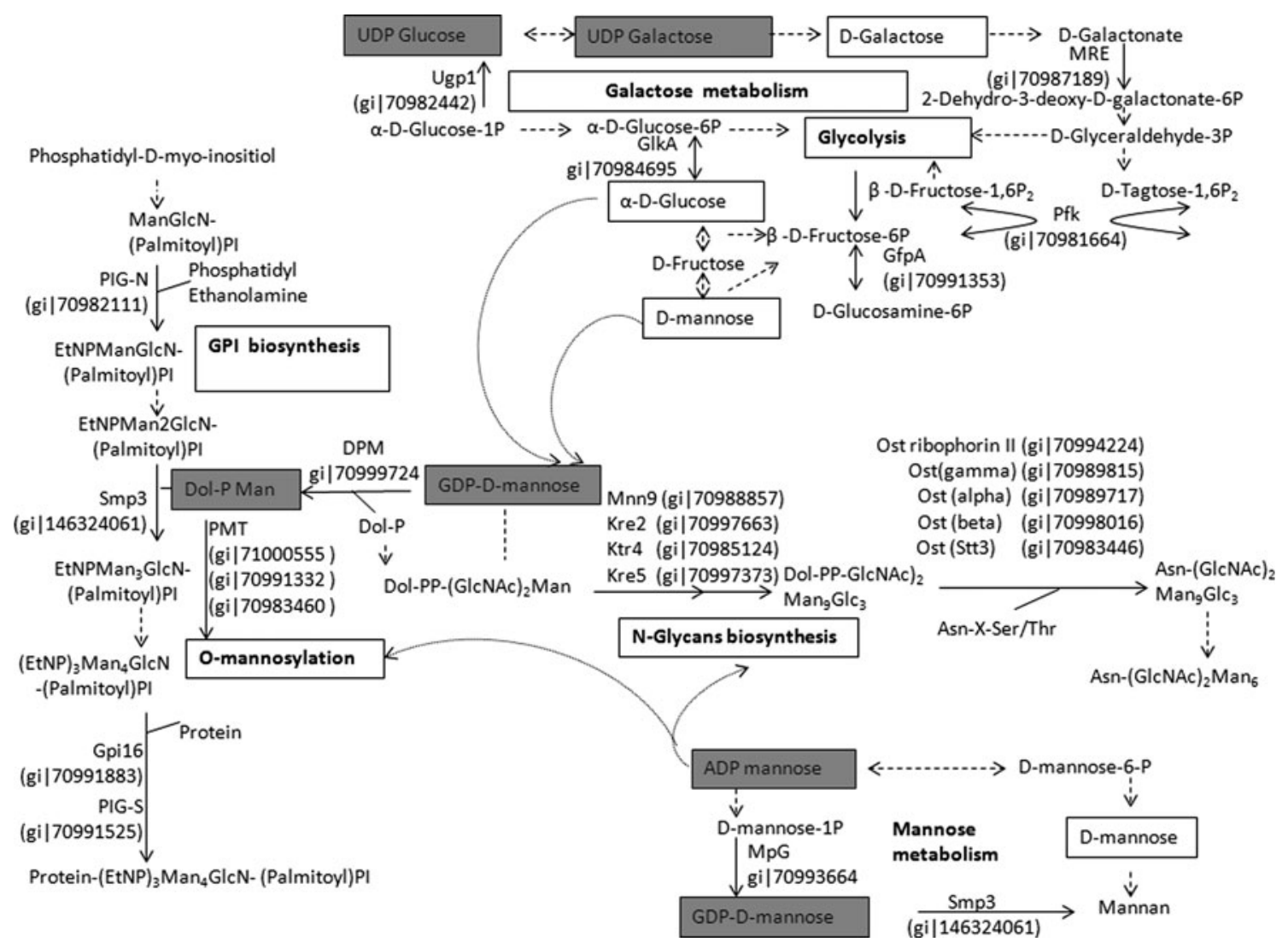

Fig. 3 Metabolic pathways of N-glycosylation, O-glycosylation, and GPI biosynthesis in A. fumigatus. Glycolysis, mannose metabolism, and galactose metabolism provide sugar monomers including GlcNAc, Man, galactose, fructose, and Glu for the biosynthesis of $\mathrm{N}$-glycans, $\mathrm{O}$-glycans, and GPI. The proteins involved are detailed in

specific steps of individual metabolic pathways. The activated donors of monosaccharides are indicated with gray background. Dolichylphosphate-mannose, which is generated from mannose metabolism, is used as donor of mannoses in GPI biosynthesis and O-mannosylation 


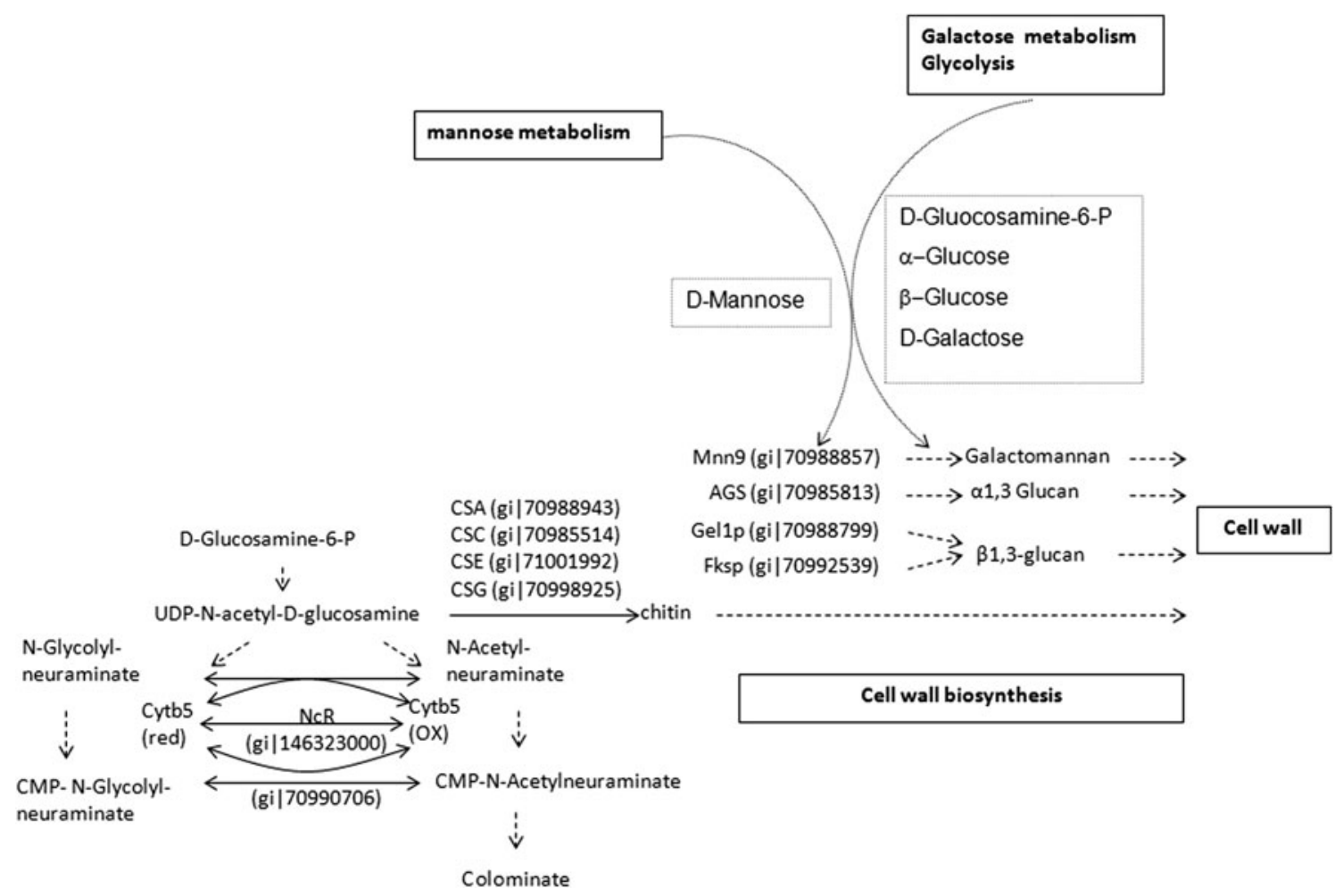

Fig. 5 Diagram of cell wall biosynthetic pathway. D-Glucosamine$6 \mathrm{P}$ generated in glycosis is transformed to UDP-GlcNAc in aminosugars metabolism and used as sugar donors for chitin biosynthesis.

Glycosis and galactose metabolism provide Gal and Man for galactomannan biosynthesis, $\alpha$-glucose for $\alpha$-glucans biosynthesis, and $\beta$-glucose for $\beta$-glucan biosynthesis 